\title{
Biochar amendments improve licorice growth and nutrient uptake through altering the root system and soil enzyme activities in loamy sand under salt stress
}

\author{
Dilfuza Egamberdieva ${ }^{1,2}$, Zohreh Zoghi ${ }^{3}$, Aida Kistaubayeva ${ }^{2}$, Ma Hua ${ }^{1,4}$, Stephan Wirth ${ }^{1}$ and \\ Sonoko Dorothea Kimura ${ }^{1,5}$ \\ ${ }^{1}$ Leibniz Centre for Agricultural Landscape Research (ZALF), Müncheberg, Germany \\ ${ }^{2}$ Department of Biotechnology, Al-Farabi Kazakh National University, 050040, Almaty, Kazakhstan \\ ${ }^{3}$ Department of Forestry, Faculty of Natural Resources and Marine Sciences, Tarbiat Modares University, \\ Noor, Mazandaran, Iran \\ ${ }^{4}$ School of Life Sciences, Chongqing University, Chongqing 401331, China \\ ${ }^{5}$ Faculty of Life Sciences, Humboldt University of Berlin, Berlin, Germany \\ * Correspondence: Dilfuza.Egamberdieva@zalf.de
}

\begin{abstract}
Licorice (Glycyrrhiza uralensis Fish.) is considered as salt and drought tolerant leguminous plant. We hypothesized that the biochar amendment into the soil might alleviate salt stress in licorice by improving its plant growth, nutrient acquisition. The present study was designed to determine the effect of biochar on licorice plant growth, acquisition of $\mathrm{C}$ (carbon), nitrogen $(\mathrm{N})$, and phosphorus (P) and on soil fluorescein diacetate (FDA) and enzyme activities under saline soil condition. Pyrolysis char from maize at 2, 4, and $6 \%$ concentrations were used for pot experiments. The shoot and/or root biomass of licorice grown in soil amended with 2 and $4 \%$ MBC under nonsaline and saline conditions was increased. The root architectural traits, like root length, surface area, project area, and root volume, as well as nodulation traits, were also significantly modified by biochar application at both 2 and $4 \%$ concentrations. The concentrations of $\mathrm{N}$ and $\mathrm{K}$ in plant tissue were increased under 2 and $4 \% \mathrm{MBC}$ amendment compared to plants grown without biochar application. Moreover, soil amended with biochar showed a positive effect on soil hydrolase activities. This study demonstrated the beneficial effects of biochar from maize on growth and nutrient uptake of licorice by improving the nodule formation, root architecture, and soil biological activity in saline soil conditions.
\end{abstract}

Keywords: biochar; licorice; soil enzymes; salinity; nutrients; root 